\title{
Routing Update Period in Cognitive Radio Ad Hoc Networks
}

\author{
Angela Sara Cacciapuoti*†, Member, IEEE, Marcello Caleffi* ${ }^{* \dagger}$, Member, IEEE, Francesco Marino*, \\ Luigi Paura*† Member, IEEE \\ *Department of Electrical Engineering and Information Technologies (DIETI) \\ University of Naples Federico II \\ Naples, Italy \\ ${ }^{\dagger}$ Laboratorio Nazionale di Comunicazioni Multimediali (CNIT) \\ Naples, Italy \\ Email:\{angelasara.cacciapuoti, marcello.caleffi, francesco.marino, paura\}@unina.it
}

\begin{abstract}
In this paper, we address the choice of the routing update period in Cognitive Radio Ad Hoc Networks with the objective of maximizing the capacity available at an arbitrary node acting as source. To this aim, first, the problem of the routing update period is reformulated to account for the slotted nature of the Cognitive Radio time induced by the spectrum sensing functionality. Then, we analytically derive the available capacity at the source node as function of the routing update period through closed-form expressions. The mathematical analysis is carried out by adopting three different routing update strategies and two different PU activity models. The theoretical analysis is validate through numerical simulations.
\end{abstract}

Index Terms - Cognitive Radio, ad hoc, routing, update period.

\section{INTRODUCTION}

Cognitive Radio (CR) paradigm has been proposed as a viable solution to counteract both spectrum inefficiency and spectrum scarcity problems. The CR paradigm exploits the concept of spectrum hole: a Cognitive User (CU) is allowed to use a spectrum band licensed to a Primary User (PU) whenever it is temporarily unused. The CR paradigm can be applied to ad hoc scenarios, and the resulting networks, referred to as CR Ad Hoc Networks (CRAHNs) [1], [2], are composed by CUs that exploit the spectrum holes for establishing multi-hop communications in a peer-to-peer fashion.

To fully unleash the potentials of CRAHNs, new challenges must be addressed and solved at the network layer. In particular, an efficient network layer design able to account for the distinguishable properties of the CR paradigm is needed. In this context, the choice of the routing update period, i.e., the choice of how frequent the routing update packets must be exchanged, is crucial as described in the following.

This work was supported by the Italian government under the following grants: PON01-00744 "DRIVE-IN2: DRIVEr monitoring: technologies, methodologies, and IN-vehicle INnovative systems for a safe and ecocompatible driving", PON01-01936 "HABITAT: HarBour traffIc opTimizAtion sysTem", PON01-03096 "MC3-Care: Mobile Continuos Connected Comprehensive-Care", PON01-02425 "SIRIO-FORM Services for a wireless network infrastructure beyond 3G", and PON01-00142 "SICURFER: Innovative Technologies for Safety and Security of Railway traffic".

\section{A. Problem Statement}

Let us consider an arbitrary ad hoc network, in which each node must forward the data traffic according to its knowledge of the network topology. Whenever a node proactively maintains such a routing knowledge, routing update packets need to be periodically exchanged among the nodes. Clearly, the choice of the routing update period deeply affects the routing performance, independently of the adopted routing protocol. In fact, the shorter is the routing update period, the more accurate is the routing knowledge. However, shorter routing update periods increase the network overhead.

In a CRAHN, the choice of the routing update period is even more crucial, due to the presence of the PU activity. In fact, in case of sudden or unexpected PU activity on a route, a fast propagation of the route unavailability assures a more accurate routing knowledge [3], at the cost of additional route overhead.

In this paper, we address the choice of the routing update period with the objective of maximizing the capacity available at an arbitrary node acting as source. Specifically, first, the problem of the routing update period is reformulated to account for the slotted nature of the CR time induced by the spectrum sensing functionality (see Sec. III-A). Then, we analytically derive the available capacity at the source node as function of the routing update period through closedform expressions. The mathematical analysis is carried out by adopting three different routing update strategies and two different PU activity models. Regarding the routing update, we consider the following strategies:

1) Perfect Knowledge Strategy: the CU source has perfect knowledge of the PU activity affecting each available route. This strategy provides an upper bound of the available capacity at the source.

2) Blind Strategy: the CU source has only a priori knowledge on the average PU activity affecting each available route;

3) Update Based Strategy: the CU source periodically receives route updates about the PU activity affecting each available route. 


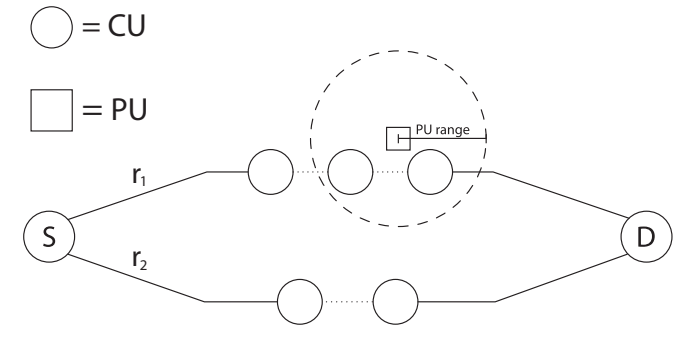

Fig. 1. Network Model.

Regarding the PU activity, we consider the following models [4], [5]:

1) Two-State Birth-Death Model: the PU activities in subsequent time slots are independent;

2) Markov Chain: the PU activities in subsequent time slots are dependent according to a Markov Chain Model.

To the best of our knowledge, no work is available in literature addressing the problem of the optimal routing update period in CR networks.

The rest of the paper is organized as follows. In Sec. II we describe the network model. In Sec. III we derive the analytical expressions of the available capacity. We evaluate the analytical results by simulations in Sec. IV. Finally, in Sec. V we conclude the paper.

\section{Network Model And Preliminaries}

In this section, we first describe the network model along with the PU activity models. Then, we collect some definitions that will be used through the paper.

\section{A. Network Model}

We consider for the sake of simplicity the network model shown in Fig. 1, where an arbitrary CU source, say $S$, can reach an arbitrary CU destination, say $D$, through two different routes: $r_{1}$ and $r_{2}$. Route $r_{1}$, affected by PU activity, offers the capacity $C_{1}$, while route $r_{2}$, unaffected by PU activity, offers the capacity $C_{2}$ with $C_{2}<C_{1}{ }^{1}$.

As well known, the $\mathrm{CU}$ activity is organized into fixed-sized slots of duration $T$. Each time slot $T$ is further organized in a sensing period $T_{s}$, which measures the portion of the time slot assigned to the spectrum sensing, and in a transmission period $T_{t x}$, which measures the portion of the time slot devoted to the $\mathrm{CU}$ packet transmissions. If the PU affecting a route is active in a certain time slot, then the route is unavailable for $\mathrm{CU}$ transmissions in such a time slot. Hence, the corresponding capacity in such a time slot is zero.

\section{B. PU Activity Model}

1) Two-State Birth-Death Model: The PU activity is modeled as a two-state birth-death process [4], with death rate $\alpha$ and birth rate $\beta$. In the on state, the PU is active with probability $P_{1}=\beta /(\alpha+\beta)$, whereas in the off state it is inactive with probability $P_{0}=1-P_{1}$.

\footnotetext{
${ }^{1}$ We can assume without loss of generality that $C_{2}<C_{1}$ since, if $C_{2} \geq$ $C_{1}$, then the choice between the two routes is obvious,
}

2) Markov Chain Model: As in [5], the on state and the off state durations are modeled as continuos random variables, independent each other and exponentially distributed with parameters $\lambda_{\mathrm{ON}}$ and $\lambda_{\mathrm{OFF}}$. Due to the slotted nature of the CR time (see Sec. III-A), the PU activity behavior collapses in a Markov chain. Therefore, If we denote with $P_{10}$ the probability of the PU being inactive in a time slot of duration $\mathrm{T}$ given that it was active in the previous time slot, we have $P_{10}=P_{0}+P_{0} e^{-\left(\lambda_{\mathrm{ON}}+\lambda_{\mathrm{OFF}}\right) T}[5]$ and $P_{11}=1-P_{10}$. Similarly, we have: $P_{00}=P_{0}+P_{1} e^{-\left(\lambda_{\mathrm{ON}}+\lambda_{\mathrm{OFF}}\right) T}$ and $P_{01}=1-P_{00}$.

\section{Definitions and Assumptions}

Definition 1. (Routing Update Period) The routing update period $\tau(K)$ is the time interval between the reception of two route update packets:

$$
\tau(K)=K \cdot T
$$

Remark. The routing update period $\tau(K)$ being a multiple of the time slot $T$ allows us to account for the slotted nature of the CR time induced by the spectrum sensing functionality.

Remark. The lower is $K$, i.e., the more frequent are the route update packets, the more accurate is the routing knowledge at the source. However, the lower is $K$, the more is the network overhead. Therefore, the proper choice of the parameter $K$ is fundamental to balance the benefits provided by an accurate routing information and the cost in terms of network overhead.

Definition 2. (Route Capacity) Given a route $r_{i}$, the route capacity $C_{i}$ is as the maximum bit-rate provided by the route in absence of both PU activity and route overhead:

$$
C_{i}=\min _{e_{j} \in r_{i}}\left\{C_{j}\right\}
$$

where $e_{j}$ is an arbitrary link of the route $r_{i}$ characterized by the capacity $C_{j}$.

Definition 3. (Route Overhead) The route overhead $\Omega(K)$ is the bit-rate required to update the routing information:

$$
\Omega(K)=\frac{L}{\tau(K)}=\frac{L}{K T}
$$

where $L$ is the cost associated to the reception of the routing update packet at the $\mathrm{CU}$ source, expressed in bits.

\section{Available CAPACITy}

With reference to the adopted network model, in this section we derive the capacity available at the $\mathrm{CU}$ source for each considered routing update strategy. The available capacity is derived by accounting for both the PU activity and the route overhead.

\section{A. Perfect Knowledge Strategy}

In any time slot the source is aware of the PU activity state without needing any routing update packet. Hence, the source is able to always select the route providing the highest capacity 


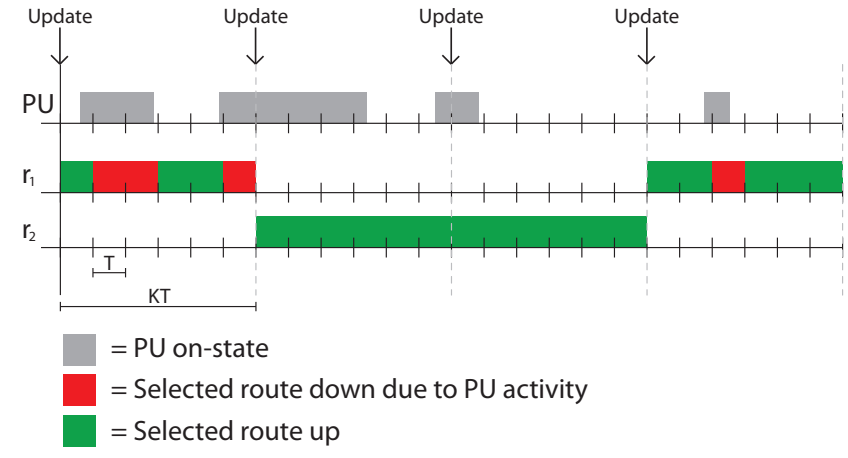

Fig. 2. Update Based Strategy: example for $\mathrm{K}=6$.

at no overhead cost. The available capacity for the considered network model is given by:

$$
C^{\mathrm{PK}}=P_{0} \cdot C_{1}+P_{1} \cdot C_{2}
$$

Remark. (4) does not depend on $K$ and it holds for both the considered PU activity models, as a consequence of the perfect knowledge strategy definition.

Remark. (4) provides an upper bound of the available capacity at the source, since: i) the source is fully aware of the PU activity in each slot; ii) the knowledge is acquired without any overhead cost.

\section{B. Blind Strategy}

The source a-priori selects the route providing the highest capacity by accounting for the average PU activity affecting the routes. Clearly, the a-priori selection does not rely on any routing update information. Hence, the available capacity for the considered network model is given by:

$$
C^{\mathrm{B}}=\max \left\{P_{0} \cdot C_{1}, C_{2}\right\}
$$

Remark. (5) does not depend on $K$ and it holds for both the considered PU activity models, as a consequence of the blind strategy definition. Moreover, it does not depend on the route overhead $\Omega(K)$.

Remark. The blind strategy should be adopted whenever the routing update information is not available.

\section{Update Based Strategy}

At the beginning of each routing update period, the source is updated on the PU activity in the current time slot through

TABLE I

UPDATE BASED AVAILABLE CAPACITy FOR $K=3$.

\begin{tabular}{|l|ll|c|}
\hline \multicolumn{3}{|c|}{ PU Activity States on $r_{1}$} & Available Capacity \\
\hline $\mathbf{0}$ & 0 & 0 & $C_{1}-\Omega(3)$ \\
$\mathbf{0}$ & 0 & 1 & $2 / 3 C_{1}-\Omega(3)$ \\
$\mathbf{0}$ & 1 & 0 & $2 / 3 C_{1}-\Omega(3)$ \\
$\mathbf{0}$ & 1 & 1 & $1 / 3 C_{1}-\Omega(3)$ \\
$\mathbf{1}$ & 0 & 0 & \\
$\mathbf{1}$ & 0 & 1 & \}$C_{2}-\Omega(3)$ \\
$\mathbf{1}$ & 1 & 0 & \} \\
$\mathbf{1}$ & 1 & 1 & \\
\hline
\end{tabular}

a routing update packet. Hence, the source selects the route providing the highest capacity by accounting for the instantaneous PU activity, i.e., the PU activity affecting the routes in the first time slot composing the routing update period. The selected route will be used in all the subsequent time slots, until a new routing update packet will be received. Clearly, if the selected route is affected by PU activity in any of the subsequent time slots, then the route fails in such time slots.

Fig. 2 shows an example of the update based strategy in the time domain. In the figure, we report the PU activity state for each time slot, along with the route chosen by the source according to the received routing update packet. More in detail, in the first routing update period the source selects route $r_{1}$ since: i) $r_{1}$ provides the highest route capacity; ii) the routing update packet reports the PU state on $r_{1}$ as off in the first time slot. We note that, since in the second, third and sixth time slot of the first routing update period the PU is on, the available capacity at the source in such time slots is $0 \mathrm{bit} / \mathrm{s}$.

A further example is reported in Table I, where we report the available capacity for $K=3$ when the update based strategy is adopted. In the table, we list all the $2^{K}$ PU state combinations, and for each combination we report the available capacity at the source.

We derive now the closed-form expression of the available capacity for both two-state birth-death PU activity model (Theorem 1) and markov chain PU activity model (Theorem 2).

Theorem 1. (Available Capacity: two-state birth-death model) By adopting the update based strategy, when the $P U$ activity is modeled as a two-state birth-death process, the available capacity $C_{\mathrm{BD}}^{\mathrm{UB}}(K)$ is:

$$
C_{\mathrm{BD}}^{\mathrm{UB}}(K)=P_{0} \cdot \frac{C_{1} \cdot\left(P_{0}(K-1)+1\right)}{K}+P_{1} C_{2}-\Omega(K)
$$

where $\Omega(K)$ is defined in (3).

Proof: The proof follows by accounting for the Bernoulli distribution.

Remark. Differently from (4) and (5), the available capacity $C_{\mathrm{BD}}^{\mathrm{UB}}(K)$ provided by the update based strategy depends not only on the PU on/off state probabilities and on the route capacities, but also on both the routing update period $\tau(K)$ and the corresponding route overhead $\Omega(K)$.

Theorem 2. (Available Capacity: Markov chain model) By adopting the update based strategy, when the PU activity is modeled as a Markov chain process, the available capacity $C_{\mathrm{MC}}^{\mathrm{UB}}(K)$ is reported in (7) at the top of the next page, where:

$$
\begin{gathered}
f\left(i, l_{1}, \ldots, l_{K-1}\right)= \begin{cases}C_{1} \frac{1+\sum_{j=1}^{K-1}\left|l_{j}-1\right|}{K} & \text { if } i=0 \\
C_{2} & \text { if } i=1\end{cases} \\
l_{j}= \begin{cases}0 & \text { if the } P U \text { is on in the } j \text {-th time slot } \\
1 & \text { otherwise }\end{cases}
\end{gathered}
$$

Proof: The Theorem can be proved by constructing a tree representing the possible events for $K$ time slots, and by exploring all the branches of the tree as in [6]. 


$$
C_{\mathrm{MC}}^{\mathrm{UB}}(K)=\sum_{i=0}^{1} P_{i} \sum_{l_{K-1}=i}^{|i-1|} P_{i l_{K-1}} \sum_{l_{K-2}=i}^{|i-1|} P_{l_{K-1} l_{K-2}} \ldots \sum_{l_{1}=i}^{|i-1|} P_{l_{2} l_{1}} f\left(i, l_{1}, \ldots, l_{K-1}\right)-\Omega(K)
$$

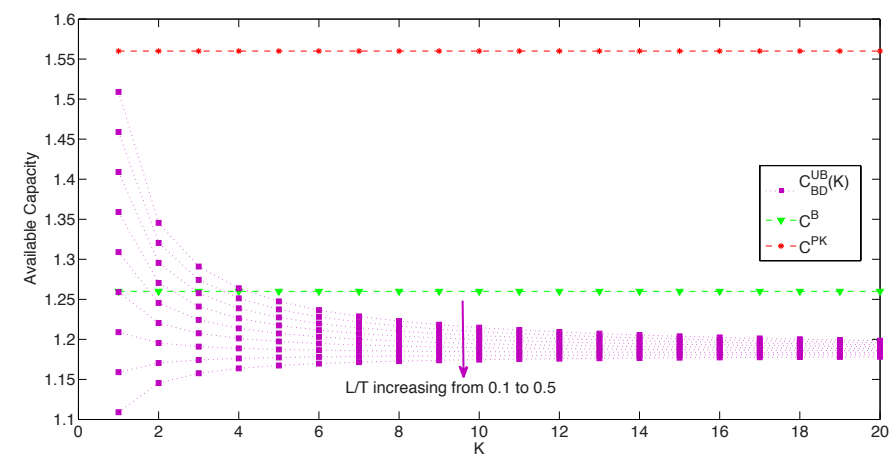

Fig. 3. Available Capacity Update Based Strategy and birth-death PU Activity.

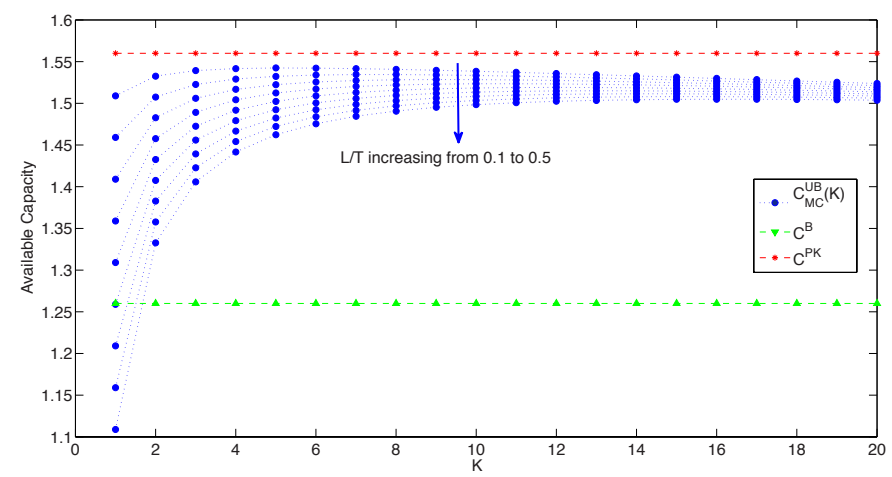

Fig. 4. Available Capacity Update Based Strategy and Markov Chain PU Activity.

Remark. Differently from (6), when the PU activity is modeled as a Markov chain, the available capacity $C_{\mathrm{MC}}^{\mathrm{UB}}(K)$ depends also on the PU activity pattern through the PU transition probabilities $P_{l_{m} l_{m-1}}$.

\section{Performance Analysis}

In this section, we validate the theoretical analysis through numerical simulations.

In all the considered experiments, the simulation setting is as follows: the PU off probability $P_{0}=0.7$, the time slot $\mathrm{T}=$ 0.01 , the normalized route update packet size $L / T \in[0.1,0.5]$, the capacity ratio $C_{1} / C_{2}=1.8$, and the sum of the PU activity duration parameters $\lambda_{\mathrm{ON}}+\lambda_{\mathrm{OFF}}=1$.

In Fig. 3, we show the behavior of the three considered strategies as a function of the route update period $K$, for the two-state birth-death PU activity model. As mentioned in Sec. III, the perfect knowledge strategy is the benchmark for the other two strategies, and both the perfect knowledge and the blind strategies do not depend on either $\mathrm{K}$ or $\Omega(K)$. The behavior of the update based strategy is deeply affected by the normalized route update packet size $L / T$. In fact, for high $L / T$ values, the higher is $K$, the better are the update based strategy performance. Differently, for low $L / T$

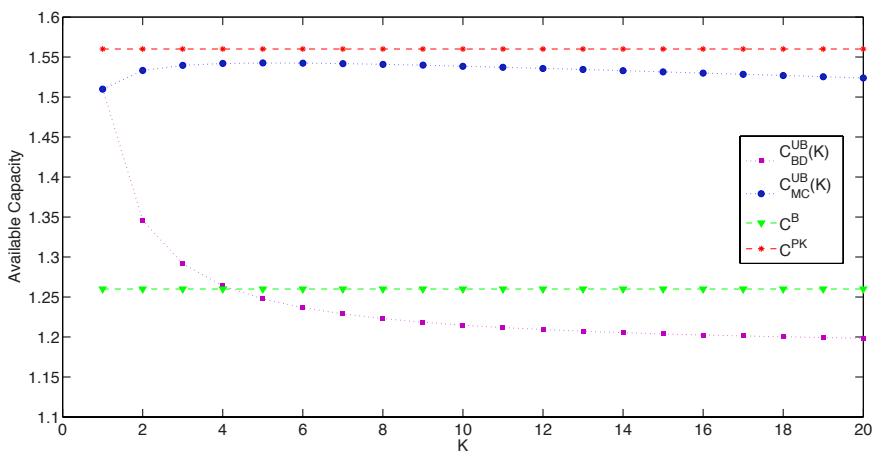

Fig. 5. Available Capacity comparison for the two PU Activity model and network knowledge.

values, the lower is $K$, the better are the update based strategy performance. Therefore, the choice of the routing update period is not univocal, but it depends on the considered scenario. We finally observe that the update based strategy is preferable with respect to the blind one only for small values of $K$ and $L / T$.

In Fig. 4, we show the behavior of the three considered strategies as a function of the route update period $K$, for the Markov chain PU activity model. All the considerations we made for the previous experiment hold also in this case. Moreover, we observe that the introduction of a dependence in the PU activity makes the update based strategy preferable with respect to the blind one for most of the considered values of $K$ and $L / T$. Finally, we observe that for any considered value of $L / T$, the update based strategy performs similarly to the benchmark whenever $K$ is not too small.

Finally, in Fig. 5, we show the deep impact of the PU activity model on the performance of the update based strategy. In particular, we observe that the higher is $K$, the more the performances of the update based strategy for the two considered PU activity models differ each other.

\section{Conclusions}

In this paper, we addressed the choice of the routing update period in Cognitive Radio Ad Hoc Networks, with the objective of maximizing the capacity available at an arbitrary node acting as source. We analytically derived the available capacity at the source node as function of the routing update period through closed-form expressions, by accounting for different routing update strategies and different PU activity models. The proposed theoretical analysis allowed us to individuate the main parameters affecting the network performance.

\section{REFERENCES}

[1] I. F. Akyildiz, W.-Y. Lee, M. C. Vuran, and S. Mohanty, "Next generation/dynamic spectrum access/cognitive radio wireless networks: A survey," COMPUTER NETWORKS, vol. 50, pp. 2127-2159, 2006. 
[2] A. Cacciapuoti, C. Calcagno, M. Caleffi, and L. Paura, "Caodv: Routing in mobile ad-hoc cognitive radio networks," in IFIP Wireless Days, 2010, pp. $1-5$.

[3] M. Caleffi, I. Akyildiz, and L. Paura, "Opera: Optimal routing metric for cognitive radio ad hoc networks," IEEE Transactions on Wireless Communications, vol. 11, no. 8, pp. 2884-2894, 2012.

[4] A. Cacciapuoti, I. Akyildiz, and L. Paura, "Optimal primary-user mobility aware spectrum sensing design for cognitive radio networks," IEEE Journal on Selected Areas in Communications, vol. PP, no. 99, pp. 112, 2013.

[5] H. Kim and K. Shin, "Efficient discovery of spectrum opportunities with mac-layer sensing in cognitive radio networks," IEEE Transactions on Mobile Computing, vol. 7, no. 5, pp. 533-545, 2008.

[6] A. Cacciapuoti, M. Caleffi, and L. Paura, "A theoretical model for opportunistic routing in ad hoc networks," in ICUMT '09: International Conference on Ultra Modern Telecommunications Workshops, 2009, pp. 1-7. 